\title{
Measurements of 3D Silicon Strip Sensors by Two Manufacturers
}

Michael Köhler*, Simon Eckert, Karl Jakobs, Susanne Kühn, Gregor Pahn, Ulrich Parzefall

University of Freiburg, Germany

E-mail: Michael.Koehlerdcern.ch

Celeste Fleta, Giulio Pellegrini, Manuel Lozano

Centro Nacional de Microelectronica (CNM-IMB-CSIC), Barcelona, Spain

Gian-Franco Dalla Betta, Andrea Zoboli

University of Trento and INFN sez. Trento, Italy

Maurizio Boscardin, Sabina Ronchin, Nicola Zorzi

Fondazione Bruno Kessler (FBK-IRST), Trento, Italy

Jaakko Härkönen, Panja-Riina Luukka, Teppo Mäenpää, Henri Moilanen

Helsinki Institute of Physics, Finland

Richard Bates, Sarah Houston, Chris Parkes

University of Glasgow, UK

The increase of the radiation dose at the luminosity upgrade of the LHC (sLHC) necessitates the development of novel tracking detectors. Among these, 3D silicon detectors constitute a promising option. By etching columnar electrodes of both doping types into the sensor, the distance for depletion and charge collection is decoupled from the sensor thickness and can be considerably smaller than in standard planar sensors. Two of the main effects of radiation damage in silicon detectors (increasing depletion voltage and trapping) can be significantly reduced.

Silicon strip detectors in 3D-DDTC (double-sided, double type column) design produced by FBKIRST (Trento, Italy) and CNM-IMB (Barcelona, Spain) have been measured in a test beam at the CERN SPS. Important properties like spatially resolved charge collection and detection efficiency were investigated.

9th International Conference on Large Scale Applications and Radiation Hardness of Semiconductor Detectors

30 September - 2 October 2009

Florence, Italy

\footnotetext{
* Speaker.
} 


\section{Introduction}

The planned Phase II upgrade of the LHC, the sLHC [U], will deliver a ten times higher peak luminosity than the LHC. After collecting an integrated luminosity of $3000 \mathrm{fb}^{-1}$, the fluence expected for the detectors in the foreseen pixel region of the LHC experiments will be in the order of $10^{16} \mathrm{Neq} / \mathrm{cm}^{2}$ and in the strip region in the order of $10^{15} \mathrm{Neq} / \mathrm{cm}^{2}$. Apart from other requirements, the unprecedented high radiation damage in the silicon sensors demands the development of new tracking detectors. One approach for radiation tolerant detectors is the 3D design [व], where columnar electrodes are etched into the sensor and doped to form junction electrodes and ohmic electrodes. The distance for charge collection and depletion is given by the spacing of the columns rather than by the entire sensor thickness as in planar sensors with electrodes on the front and the back surface, see Fig. $\mathrm{W}$. A short distance between the columns compared to the sensor thickness results in lower depletion voltage and reduces trapping effects. This is especially beneficial for irradiated detectors to reduce the increase of the depletion voltage with radiation and to reduce the signal loss due to radiation-induced trapping.
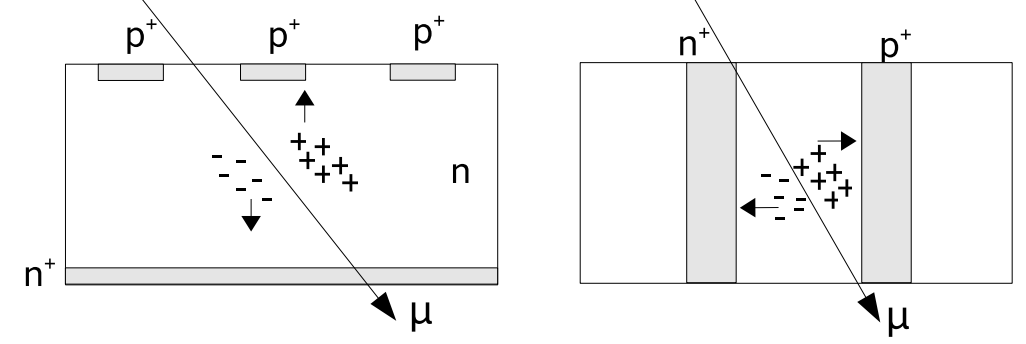

Figure 1: Principle of a standard planar silicon sensor (left, here n-type bulk) and a 3D sensor (right) with columnar electrodes passing through the entire substrate.

Compared to the fabrication of planar silicon sensors, the processing technology of 3D sensors is more complex and requires a higher number of processing steps. To simplify the fabrication process and to develop a technology which is suitable for large-scale productions, the 3D-DDTC

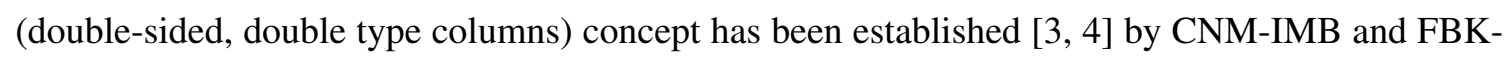
IRST. These detectors are fabricated in a double-sided process and have columns which do not pass through the entire detector thickness. This article presents results of test beam measurements performed with 3D-DDTC detectors at the CERN SPS.

\section{Devices Under Test and Test Beam Setup}

The devices under test are 3D-DDTC strip detectors produced by CNM-IMB and FBK-IRST on n-type substrate. Both devices originate from the first batch ever produced in the respective institute. A schematic cross section of the CNM design for pixel detectors is shown in Fig. \, the FBK design is similar with some modifications. The sensor properties are detailed in Table $\mathbb{}$. Junction columns ( $\mathrm{p}^{+}$doped) are etched from the front side into the wafer and ohmic columns $\left(\mathrm{n}^{+}\right)$ from the back side. The columns are partially filled with poly-silicon in the CNM design and are 
left unfilled in the FBK design. To form strip detectors, the junction columns are connected by means of a metallisation. The FBK sensors have an additional $\mathrm{p}^{+}$-doped trace below the metal. Both detectors have DC coupled readout pads and the FBK detectors have additional readout pads with AC coupling. A further difference between the sensors of both manufacturers is related to the contact on the back surface: it is covered by a uniform $\mathrm{n}^{+}$doping layer and a metallisation in the FBK sensors, whereas an oxide layer covers the back surface of the CNM sensors followed by a layer of poly-silicon and metallisation. All ohmic columns are shorted together in both designs. Further details of the detector layouts can be found in [四] and [䧃]. Full depletion of both devices is achieved below $10 \mathrm{~V}$ bias.

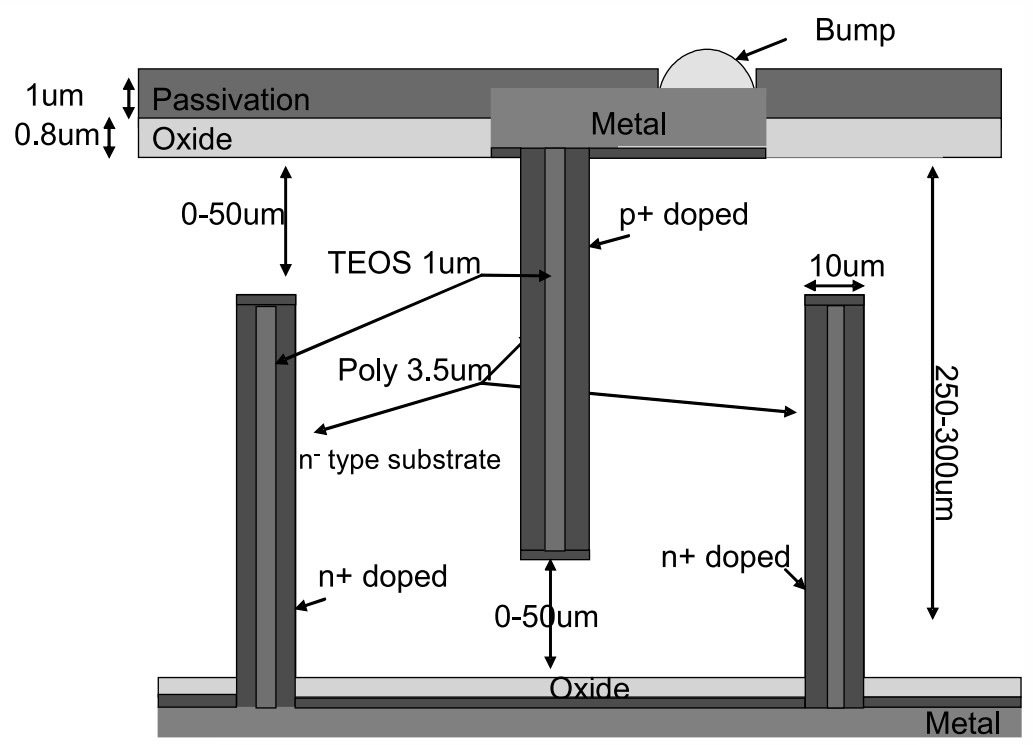

Figure 2: Schematic cross section of the CNM 3D-DDTC pixel detector design [B]. The detectors in strip layout investigated in this article have the same cross section apart from the bump bond connection which is missing for strip detectors.

\begin{tabular}{ccc}
\hline Property & 3D-DDTC (FBK) & 3D-DDTC (CNM) \\
\hline Thickness & $(300 \pm 15) \mu \mathrm{m}$ & $(285 \pm 15) \mu \mathrm{m}$ \\
Strip pitch / inter column pitch & $100 \mu \mathrm{m}$ & $80 \mu \mathrm{m}$ \\
Strip length & $8.1 \mathrm{~mm}$ & $4.0 \mathrm{~mm}$ \\
Number of strips & 81 & 50 \\
Depth of junction / ohmic columns & $190 \mu \mathrm{m} / 160 \mu \mathrm{m}$ & $250 \mu \mathrm{m} / 250 \mu \mathrm{m}$ \\
Column diameter & $\sim 10 \mu \mathrm{m}$ & $\sim 10 \mu \mathrm{m}$ \\
\hline
\end{tabular}

Table 1: Design properties of the two detectors under test.

The test beam measurements were performed with $225 \mathrm{GeV}$ pions at the $\mathrm{H} 2$ beam line of the CERN SPS. The Silicon Beam Telescope (SiBT) [5] with CMS data acquisition hardware was used to determine the reference tracks. The track impact position on the devices under test is 
known with a resolution of approximately $4 \mu \mathrm{m}$. The detectors were connected to CMS APV25 front-end chips [ [6] with $50 \mathrm{~ns}$ peaking time and analogue readout. Details on the reconstruction of the telescope reference tracks can be found in [ [ $]$. The telescope reference sensors and the devices under test were positioned perpendicularly to the beam. While the entire surfaces of both detectors were illuminated by the pion beam, the focus of the beam was centred onto the FBK sensor. Since the active surface of the FBK sensor $(8.1 \mathrm{~mm} \times 8.1 \mathrm{~mm})$ is approximately a factor of four larger than the active surface of the CNM sensor $(4 \mathrm{~mm} \times 4 \mathrm{~mm})$, a considerably higher number of tracks was recorded with the FBK detector.

\section{Results}

The conversion of the detector signals, measured in ADC counts, into charge was performed using the signal spectrum of the telescope reference detectors. The distributions of the signals measured with the telescope detectors were fitted with a convolution of a Landau function and a Gaussian. Since a perfect charge yield is expected for these detectors, the most probable value of the Landau distribution was equated with the charge expected for the given thickness of silicon. In $300 \mu \mathrm{m}$ silicon a charge of 23000 electrons ( $3.7 \mathrm{fC}$ ) is expected.

Measurements with bias voltages of up to $40 \mathrm{~V}$ and $24 \mathrm{~V}$ were performed with the FBK and CNM sensors, respectively. The maximum voltages have been chosen in order to apply bias voltages well above depletion on the one hand, and to keep the leakage current below a safe limit on the other hand.

The quantitative investigations (like maximum signal and efficiency) presented below use the data measured in the runs with the highest voltages. However, only low statistics has been acquired with the CNM detector at a bias voltage of $24 \mathrm{~V}$. Therefore, the investigations of the space resolved signal measured with the CNM detector, which requires high statistics, was performed using data measured with a bias voltage of $9 \mathrm{~V}$. For the CNM detector the highest amount of tracks was measured at this bias voltage.

\subsection{Collected Charge}

Figure 3 shows a two-dimensional representation of the signal measured with the CNM detector at $9 \mathrm{~V}$. For the illustration, the entire sensor area has been superimposed onto one unit cell with the junction column in the centre and one quarter of an ohmic column in each corner. Then, this unit cell has been divided into bins of $5 \mu \mathrm{m} \times 5 \mu \mathrm{m}$ and for each of these bins the mean signal has been calculated. The signals of two channels adjacent to the track expected impact positions are summed up to eliminate effects of charge sharing between neighbouring readout strips. In both detectors investigated, the signal measured in the column regions is lower than in the rest of the sensor since the columns constitute passive volume. However, as the columns do not pass through the sensor completely, the energy deposited below or above the columns can be measured.

When restricting to tracks impinging far away from the columns, the signal distribution shows a Landau-like spectrum. Figure $\$$ displays the signal spectrum of both devices under test for tracks with a distance of at least $25 \mu \mathrm{m}$ to a column centre. As above, the signals of two channels adjacent to the track expected impact position are summed up to avoid a broadening of the spectrum due to charge sharing. The Landau distribution most probable value (MPV) is extracted from a fit of a 


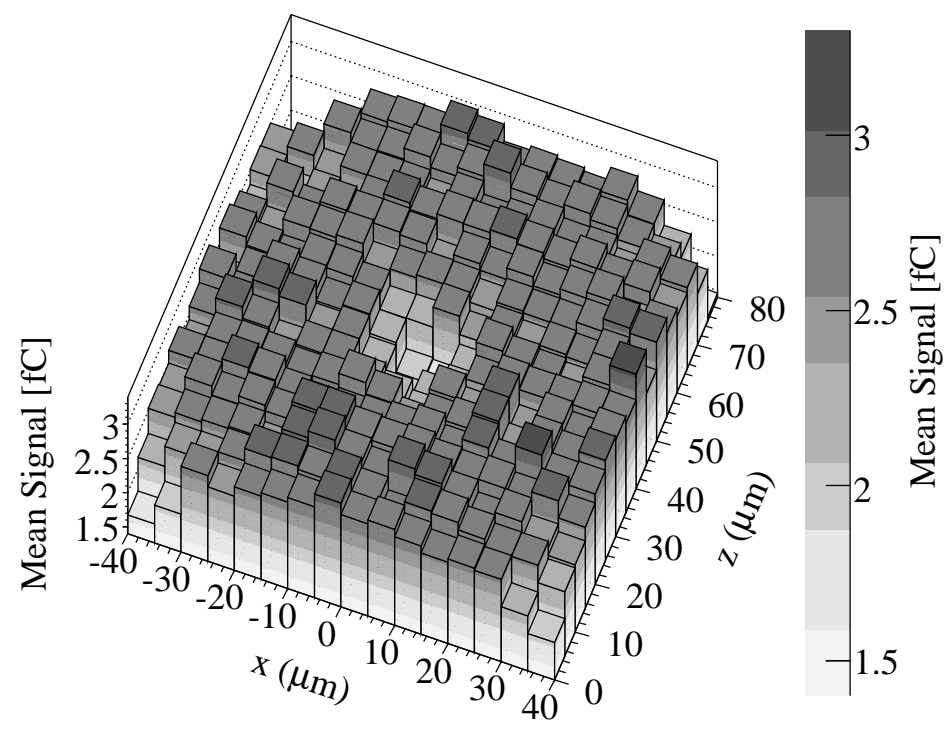

Figure 3: Mean signal in a unit cell of the CNM detector measured at $9 \mathrm{~V}$. The signals of two strips closest to the track expected impact position are summed up. The junction column is located in the centre, the readout strip is running parallel to the $z$-axis around $x=0 \mu \mathrm{m}$.
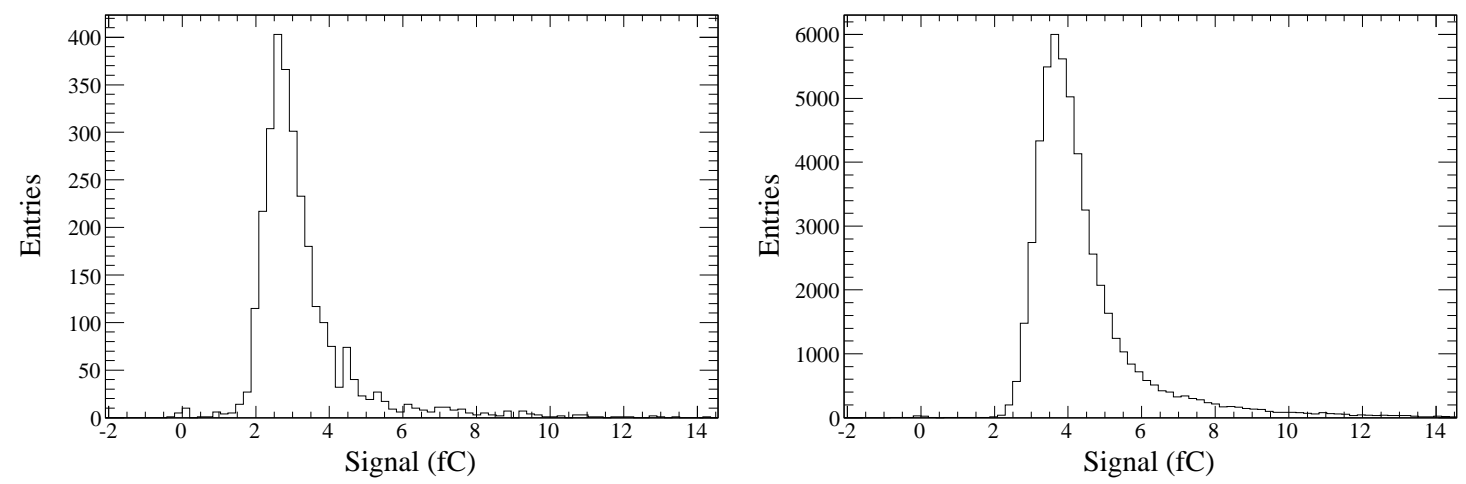

Figure 4: Signal spectrum of the CNM detector (24 V bias, left, with Landau MPV=(2.5 \pm 0.2$)$ fC) and of the FBK detector $(40 \mathrm{~V}$ bias, right, with Landau MPV= $(3.5 \pm 0.3) \mathrm{fC}$ ) for tracks hitting the sensor at least $25 \mu \mathrm{m}$ away from the centre of a column. Signals of the two strips closest to the track expected impact position are summed up. As a lower number of tracks was recorded with the CNM sensor, the left plot contains lower statistics.

convolution of a Landau function and a Gaussian to the spectrum. For the FBK sensor, the most probable signal at $40 \mathrm{~V}$ bias is $(3.5 \pm 0.3) \mathrm{fC}$, which is in agreement with the expected signal of $(3.7 \pm 0.2)$ fC for the thickness of $(300 \pm 15) \mu \mathrm{m}$. A noise of $(0.113 \pm 0.009) \mathrm{fC}$, corresponding to $(710 \pm 50)$ electrons, was measured. This corresponds to a signal-to-noise ratio of $\sim 31$. The most probable signal determined for the CNM detector at $24 \mathrm{~V}$ bias is $(2.5 \pm 0.2) \mathrm{fC}$ and is thus approximately $30 \%$ lower than the expected value of $(3.5 \pm 0.2)$ fC for a $(285 \pm 15) \mu \mathrm{m}$ thick 
silicon sensor. The reason for this signal loss is so far not explained, but it is attributed to imperfections specific to the batch this detector originates from. For the CNM sensor the noise was $(0.083 \pm 0.006) \mathrm{fC}$, corresponding to $(520 \pm 40)$ electrons, which yields a signal-to-noise ratio of $\sim 30$. Although the signal measured with the CNM sensor is lower than that of the FBK sensor, the signal to noise ratio is approximately the same in both sensors. This is attributed to the shorter strip length in the CNM sensor which leads to lower capacitance and results in lower noise.

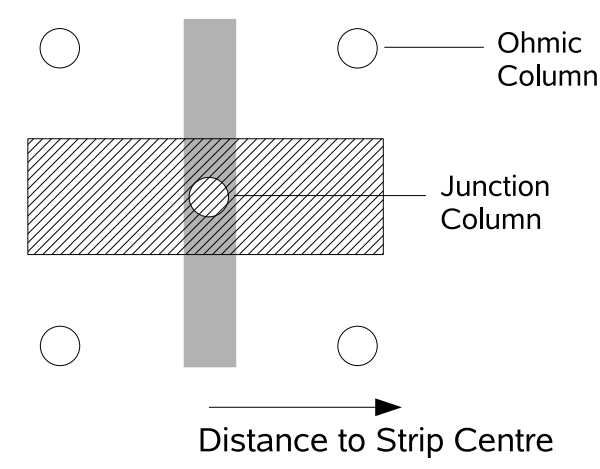

Figure 5: Sketch of the unit cell with the junction column in the centre. The grey shaded area represents the vertically running readout strip. Tracks impinging on the hatched region, which has a width of $30 \mu \mathrm{m}$ in direction of the readout strips, are used for the investigation of mean charge versus distance to strip centre shown in Fig. 6.

The electric field reaches a minimum in the middle of two neighbouring readout strips. In the sketch of the unit cell, shown in Fig. [, these critical regions are located at the left and right margins. To investigate the uniformity of the signal in the regions with reduced electric field, the mean signal is plotted versus the distance to the strip centre in Fig. 6. Only tracks impinging in the hatched region of Fig. 1 are used, therefore tracks depositing lower charge in the regions of the back columns are excluded. The mean signal has been favoured over the Landau most probable value since the signal spectrum for bins bordering the columns does not show a clear Landau-like shape. In those bins a significant number of entries coming from tracks which go directly through the columns lead to distortions of the Landau spectra. Since mean values are shown in Fig. 6 , these values are higher than the Landau most probable values reported above. Although the charge sharing is reduced in 3D detectors compared to planar sensors [ [8] owing to the field configuration, the effect is still visible. The charge generated by single strip signals drops visibly in the middle between two readout strips. When summing up the signals of the two strips adjacent to the track reference point, this signal drop vanishes. Thus, the total collected charge is not reduced in the region with low electric field. The only drop that remains, around the centre of the strip, is caused by the passive volume inside the junction column. It can therefore be concluded that the total collected charge is uniform across the entire sensor area (apart from the column positions). This is an improvement compared to 3D-STC (single type column) detectors with columns of the same doping type etched from only one type into the sensor. In detectors of this type, which can be regarded as the predecessor technology of 3D-DDTC detectors, the signal is lower in the middle 
between the readout strips due to the field configuration [Q]
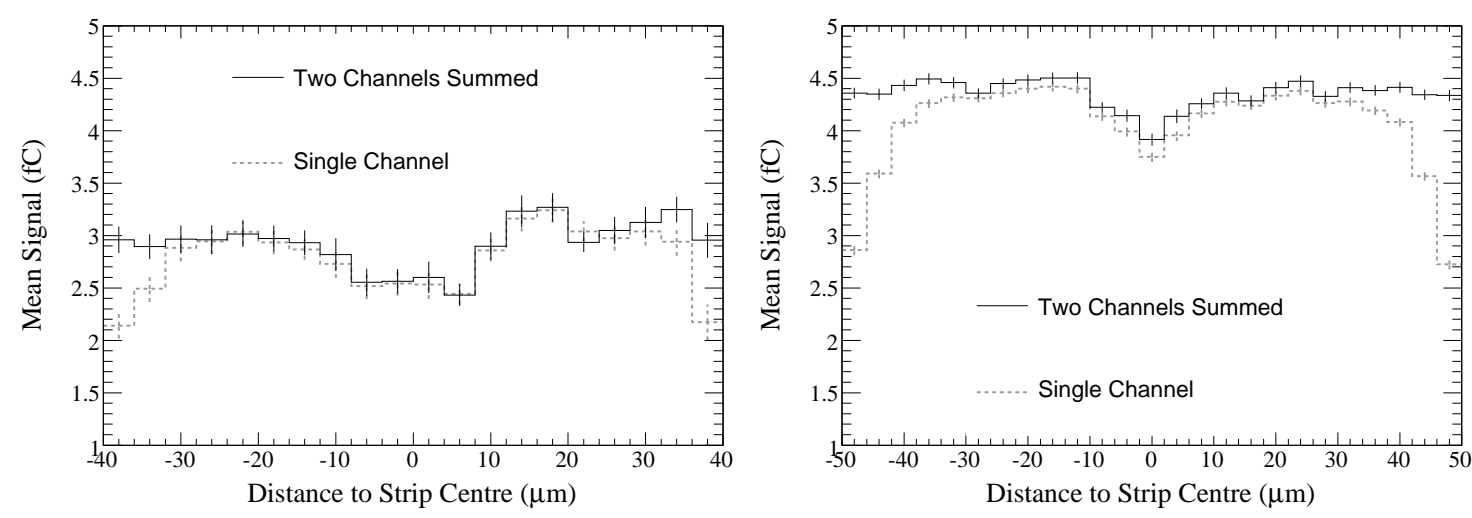

Figure 6: Mean signal versus distance to strip centre for the CNM sensor at $24 \mathrm{~V}$ bias (left) and for the FBK sensor at $40 \mathrm{~V}$ bias (right). Only tracks in the hatched region of Fig. 5 are included. Due to lower statistics, the plot for the CNM detector shows more fluctuations.

\subsection{Efficiency}

Apart from the collected charge, the efficiency is an important quantity for a characterisation of silicon detectors. For a given signal threshold, the efficiency is the fraction of events where the signal is higher than this threshold. The signal has been calculated in two different ways: using single strip signals or using the sum of the signals in two neighbouring strips. When using single strip signals, the highest signal in the strip closest to the track expected impact position or one of its neighbours was used. When using the sum of two neighbouring strips, the signals in the two strips closest to the track expected impact position were summed up. Only tracks pointing to a position well away from the sensor edges were included. The efficiency as a function of the signal threshold is shown in Fig. W. It has to be noted that the lower signal in the CNM detector (as presented above)
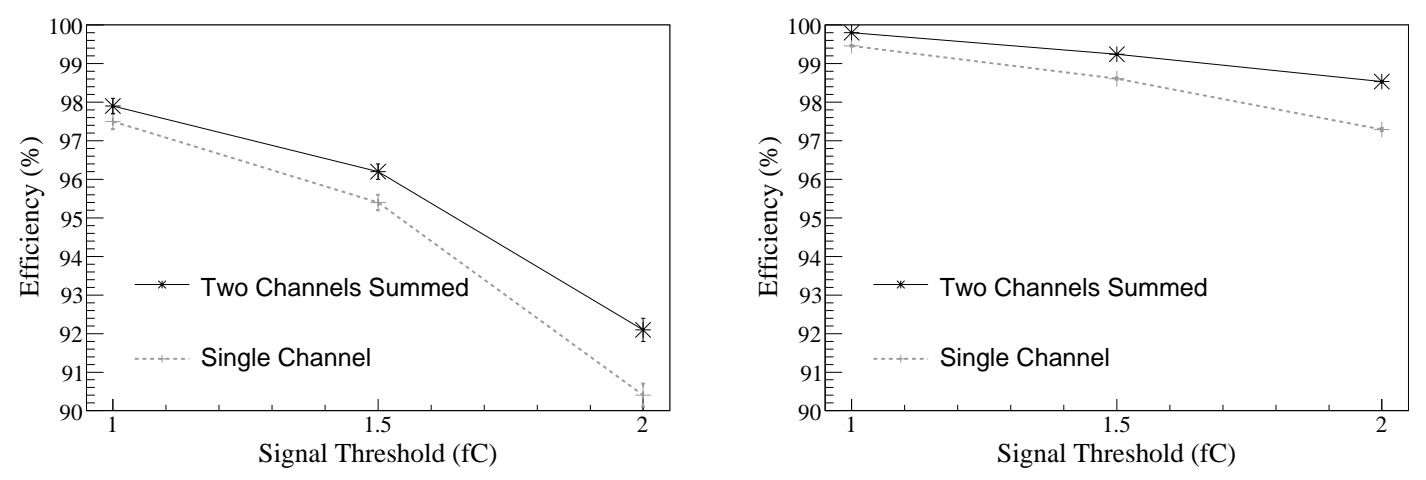

Figure 7: Efficiency for three different thresholds of the CNM sensor (left, $24 \mathrm{~V}$ bias) and the FBK sensor (right, $40 \mathrm{~V}$ bias). The values have been calculated using signals of single strips and the sum of two strips adjacent to the hit position, respectively. 
leads to a lower efficiency compared to the FBK sensor. In addition, the efficiency of the CNM sensor is reduced by the higher amount of inactive volume due to the larger column depth and the smaller pitch, which results in a higher fraction of the sensor covered with columns. The effect of charge sharing is visible as the difference between the two curves, showing the efficiency of single strips and the sum of the signals of two neighbouring strips. At a signal threshold of $1 \mathrm{fC}$ and with the signals of two neighbouring strips summed up, the efficiency reaches $(97.9 \pm 0.2) \%$ for the CNM detector and $(99.80 \pm 0.01) \%$ for the FBK detector.

\section{Conclusion}

The test beam measurements performed with 3D-DDTC detectors originating from the first batch produced at CNM and FBK, respectively, prove the full functionality of the devices. Uniform charge collection and high efficiency of more than $97 \%$ in both sensors at $1 \mathrm{fC}$ has been measured with electronics with $50 \mathrm{~ns}$ shaping time. The radiation hardness of 3D-DDTC is currently being studied in laboratory and test beam measurements.

\section{References}

[1] F. Gianotti et al., Physics potential and experimental challenges of the LHC luminosity upgrade, Eur. Phys. J., vol. C39, pp. 293-333, 2005.

[2] S. I. Parker, C. J. Kenney and J. Segal, 3D - A proposed new architecture for solid-state radiation detectors, Nucl. Instrum. Meth. A, vol. 395, pp. 328-343, 1997.

[3] G. Pellegrini, M. Lozano, M. Ullan, R. Bates, C. Fleta and D. Pennicard, First double-sided 3-D detectors fabricated at CNM-IMB, Nucl. Instrum. Meth. A, vol. 592, pp. 38-43, 2008.

[4] A. Zoboli, G. F. Dalla Betta, M. Boscardin, C. Piemonte, S. Ronchin, N. Zorzi and L. Bosisio, Double-sided, double-type-column 3-D detectors: Design, fabrication, and technology evaluation, IEEE Trans. Nucl. Sci., vol. 53, no. 5, pp. 2775-2784, 2008.

[5] T. Mäenpää et al., Silicon beam telescope for LHC upgrade tests, Nucl. Instrum. Meth. A, vol. 593, pp. 523-529, 2008.

[6] M. J. French et al., Design and results from the APV25, a deep sub-micron CMOS front-end chip for the CMS tracker, Nucl. Instrum. Meth. A, vol. 466, pp. 359-365, 2001.

[7] M. J. Kortelainen, T. Lampen, H. Moilanen and T. Mäenpää, Off-line calibration and data analysis for the silicon beam telescope on the CERN H2 beam, Nucl. Instrum. Meth. A, vol. 602, pp. 600-606, 2009.

[8] D. Pennicard et al., Charge sharing in double-sided 3D Medipix2 detectors, Nucl. Instrum. Meth. A, vol. 604, pp. 412-415, 2009.

[9] T. Ehrich et al., Laser characterisation of a 3D single-type column p-type prototype module read out with ATLAS SCT electronics, Nucl. Instrum. Meth. A, vol. 583, pp. 153-156, 2007. 